Homology, Homotopy and Applications, vol.18(2), 2016, pp.209-215

\title{
CONTINUITY OF COARSE SHAPE GROUPS
}

\author{
NIKOLA KOCEIĆ BILAN
}

\author{
(communicated by Alexander Mishchenko)
}

\begin{abstract}
We show that every coarse shape group can be obtained as the inverse limit of an inverse system of the groups coming from an $\mathrm{HPol}_{\star}$-expansion. This provides a way of computing of these interesting topological invariants (whose algebraic structure is significantly richer than those of the homotopy and shape groups) in an easier manner. It is proven that, for inverse systems of compact polyhedra, the coarse shape group functor commutes with the inverse limit.
\end{abstract}

\section{Introduction and preliminaries}

Since its foundation the main goal of algebraic topology was to obtain some functorial relations between topology and algebra. One of the most important ideas of algebraic topology is to assign certain algebraic objects to different topological spaces. This idea allows studying of isomorphisms between corresponding algebraic structures instead of topological isomorphisms (homeomorphisms, homotopy or shape equivalences). This approach has a full significance for those spaces for which it is much easier to consider algebraic properties instead of topological ones. A desirable property of such algebraic objects is non-triviality and, moreover, that they properly represent a complicated structure of some topological objects by their, sufficiently rich, algebraic structure. The homotopy group functor $\pi_{k}$, relating the pointed homotopy category $H T o p_{\star}$ and the group category Grp, provides good information on spaces having good local properties (such as: polyhedra, $C W$-complexes or, more generally, spaces having the homotopy type of $A N R$-s). Outside that class, algebraic tools of shape theory (e.g., the shape group functor $\check{\pi}_{k}$ and homotopy pro-group functor pro$\pi_{k}$ which relate the pointed shape category $S h_{\star}$ to the categories Grp and pro-Grp, respectively) are more suitable. The standard example is the Warsaw circle whose global similarity with the 1-sphere $S^{1}$ structure is not detected by homotopy theory. However, there are many spaces which neither shape theory nor its algebraic tools can treat in a sufficiently good way. The standard examples for that are solenoids whose rather complicated structure is not detected by the shape group functor, since the shape groups of solenoids vanish in all dimensions. Coarse shape theory, which functorially generalizes shape theory, was founded in $[\mathbf{5}]$ to overcome such problems. The

Received October 20, 2014, revised February 2, 2016; published on September 21, 2016.

2010 Mathematics Subject Classification: 55P55, 55Q05, 55N99.

Key words and phrases: inverse limit, inverse system, pro-category, pro*-category, expansion, shape, coarse shape, homotopy (pro-)group, coarse shape group.

Article available at http://dx.doi.org/10.4310/HHA.2016.v18.n2.a11

Copyright (C) 2016, International Press. Permission to copy for private use granted. 
category frame for this theory is the (pointed) coarse shape category $S h^{*}\left(S h_{\star}^{*}\right)$, having (pointed) topological spaces as objects and having the (pointed) shape category $S h\left(S h_{\star}\right)$ as a subcategory. There are several algebraic coarse shape invariants induced by the functors $\operatorname{pro}^{*}-\pi_{k}: S h_{\star}^{*} \rightarrow$ pro-Grp (see [4]) and $\check{\pi}_{k}^{*}: S h_{\star}^{*} \rightarrow G r p, n \in \mathbb{N}$ (see $[3])$. The functor $\check{\pi}_{k}^{*}$ assigns to every pointed space $\left(X, x_{0}\right)$ the $k$-th coarse shape group $\check{\pi}_{k}^{*}\left(X, x_{0}\right)$ having the $k$-th shape group $\check{\pi}_{k}\left(X, x_{0}\right)$ as a subgroup. For a pointed compactum, unlike the shape groups, coarse shape groups fit into a long exact sequence (see $[\mathbf{2}]$ ). Therefore the study of coarse shape groups can be very useful, especially when the corresponding shape groups vanish as for solenoids. Besides obvious advantages which coarse shape groups have over shape groups, if one compares coarse shape groups with homotopy pro-groups, it is easy to notice that studying coarse shape groups is usually more fruitful. Namely, for a pointed compactum $\left(X, x_{0}\right)$, the triviality of pro- $\pi_{k}\left(X, x_{0}\right)$ is equivalent to the triviality of $\check{\pi}_{k}^{*}\left(X, x_{0}\right)=0, k \in \mathbb{N}$, but on the other hand, pro- $\pi_{k}\left(X, x_{0}\right)$ does not have the algebraic structure of a group while $\check{\pi}_{k}^{*}\left(X, x_{0}\right)$ does. In other words, the coarse shape groups keep information which homotopy pro-groups have and which are usually lost in the inverse limit process needed to obtain shape groups. However, although coarse shape groups provide useful information on a pointed space, on the other hand, a certain disadvantage is that they are too large and it is not easy to compute them for a concrete space (excepting a polyhedron). In [1] , an explicit formula for computing coarse shape groups of a pointed compactum whose bonding homomorphisms of its homotopy pro-groups are monomorphisms is given. In the present paper we give a more general and useful formula for computing a coarse shape group of any space. It will be shown that every coarse shape group can be obtained as the inverse limit of a group inverse system (Theorem 2.1) instead of applying the original definition in a natural but rather complicated manner. Since, for inverse systems of compact polyhedra, the coarse shape group functor commutes with the inverse limit, we may use the term "continuity of the coarse shape group" (Corollary 3.1). A similar discussion about continuity, concerning the functor lim, one can find in [7] (for the coarse and weak shape categories) and in [6] (for homology and shape groups).

Let us recall some elementary notions about coarse shape (see [5]). The pointed homotopy category HTop ${ }_{\star}$ has all pointed spaces $\left(X, x_{0}\right)$ for its objects and the morphisms are all pointed homotopy classes $[f]$ of mappings of pointed spaces, $f:\left(X, x_{0}\right) \rightarrow\left(Y, y_{0}\right)$. In this paper morphisms of the category $H T o p_{\star}$ will usually be denoted by omitting the brackets. The restriction of the class of objects of $H T o p_{\star}$ to pointed polyhedra yields the full subcategory $H P o l_{\star} \subseteq H T o p_{\star}$. An $S^{*}$-morphism $\left(f, f_{\mu}^{n}\right):\left(\boldsymbol{X}, \boldsymbol{x}_{0}\right) \rightarrow\left(\boldsymbol{Y}, \boldsymbol{y}_{0}\right)$ of inverse systems $\left(\boldsymbol{X}, \boldsymbol{x}_{0}\right)=\left(\left(X_{\lambda}, x_{\lambda}\right), p_{\lambda \lambda^{\prime}}, \Lambda\right)$ and $\left(\boldsymbol{Y}, \boldsymbol{y}_{0}\right)=\left(\left(Y_{\mu}, y_{\mu}\right), q_{\mu \mu^{\prime}}, M\right)$, in the category $H T o p_{\star}$, consists of an index function $f: M \rightarrow \Lambda$, and of a set of homotopy classes $f_{\mu}^{n}:\left(X_{f(\mu)}, x_{f(\mu)}\right) \rightarrow\left(Y_{\mu}, y_{\mu}\right), n \in$ $\mathbb{N}, \mu \in M$, such that, for every related pair $\mu \leqslant \mu^{\prime}$ in $M$, there exists a $\lambda \in \Lambda$, $\lambda \geqslant f(\mu), f\left(\mu^{\prime}\right)$, and there exists an $n \in \mathbb{N}$ so that, for every $n^{\prime} \geqslant n, f_{\mu}^{n^{\prime}} p_{f(\mu) \lambda}=$ $q_{\mu \mu^{\prime}} f_{\mu^{\prime}}^{n^{\prime}} p_{f\left(\mu^{\prime}\right) \lambda}$. An $S^{*}$-morphism $\left(f, f_{\mu}^{n}\right):\left(\boldsymbol{X}, \boldsymbol{x}_{0}\right) \rightarrow\left(\boldsymbol{Y}, \boldsymbol{y}_{0}\right)$ is said to be equivalent to an $S^{*}$-morphism $\left(f^{\prime}, f_{\mu}^{\prime n}\right):\left(\boldsymbol{X}, \boldsymbol{x}_{0}\right) \rightarrow\left(\boldsymbol{Y}, \boldsymbol{y}_{0}\right)$, denoted by $\left(f, f_{\mu}^{n}\right) \sim\left(f^{\prime}, f_{\mu}^{\prime n}\right)$, provided every $\mu \in M$ admits a $\lambda \in \Lambda, \lambda \geqslant f(\mu), f^{\prime}(\mu)$, and an $n \in \mathbb{N}$, such that, for every $n^{\prime} \geqslant n, f_{\mu}^{n^{\prime}} p_{f(\mu) \lambda}=f_{\mu}^{\prime n^{\prime}} p_{f^{\prime}(\mu) \lambda}$. The category pro $^{*}-H T o p_{\star}$ has as objects all inverse systems $\left(\boldsymbol{X}, \boldsymbol{x}_{0}\right)$ of pointed spaces and as morphisms all equivalence classes 
$f^{*}=\left[\left(f, f_{\mu}^{n}\right)\right]$ of $S^{*}$-morphisms $\left(f, f_{\mu}^{n}\right)$. It is related with the (well-known) category pro-HTop $p_{\star}$ via the "inclusion" functor $\mathrm{J}$ : pro-HTop $_{\star} \rightarrow$ pro $^{*}-H T o p_{\star}$ which keeps inverse systems fixed and to each morphism $f=\left[\left(f, f_{\mu}\right)\right]$ of ${\text { pro- } H T o p_{\star}}_{\text {assigns a }}$ morphism $\underline{J}(\boldsymbol{f})$ of pro $^{*}-H T o p_{\star}$ represented by $\left(f, f_{\mu}^{n}\right), f_{\mu}^{n}=f_{\mu}, \mu \in M, n \in \mathbb{N}$.

The category $H P o l_{\star}$ is a pro-reflective subcategory of $H T o p_{\star}$ (see [6]) which means that, for every pointed space $\left(X, x_{0}\right)$, there exists an $H P_{x_{\star}}$-expansion of $\left(X, x_{0}\right)$ which is a morphism $\boldsymbol{p}=\left[\left(p_{\lambda}\right)\right]:\left(X, x_{0}\right) \rightarrow\left(\boldsymbol{X}, \boldsymbol{x}_{0}\right)=\left(\left(X_{\lambda}, x_{\lambda}\right), p_{\lambda \lambda^{\prime}}, \Lambda\right)$ of pro-HTop $p_{\star}$, where $\left(\boldsymbol{X}, \boldsymbol{x}_{0}\right)$ is an inverse system of pointed polyhedra. Let $\boldsymbol{p}:\left(X, x_{0}\right) \rightarrow$ $\left(\boldsymbol{X}, \boldsymbol{x}_{0}\right)$ and $\boldsymbol{q}:\left(Y, y_{0}\right) \rightarrow\left(\boldsymbol{Y}, \boldsymbol{y}_{0}\right)$ be $H$ Pol $_{\star}$-expansions of pointed spaces $\left(X, x_{0}\right)$ and $\left(Y, y_{0}\right)$, respectively. A pointed coarse shape morphism $F^{*}:\left(X, x_{0}\right) \rightarrow\left(Y, y_{0}\right)$ is an equivalence class $\left\langle\boldsymbol{f}^{*}\right\rangle$ which is represented by a morphism $\boldsymbol{f}^{*}:\left(\boldsymbol{X}, \boldsymbol{x}_{0}\right) \rightarrow\left(\boldsymbol{Y}, \boldsymbol{y}_{0}\right)$ of pro $^{*}-H P o l_{\star}$. The pointed coarse shape category $S h_{\star}^{*}$ has for its objects all pointed topological spaces $\left(X, x_{0}\right)$, while a morphism set $S h_{\star}^{*}\left(\left(X, x_{0}\right),\left(Y, y_{0}\right)\right)$ consists of all equivalence classes $F^{*}=\left\langle\boldsymbol{f}^{*}\right\rangle$ of morphisms $\boldsymbol{f}^{*}=\left[\left(f, f_{\mu}^{n}\right)\right]:\left(\boldsymbol{X}, \boldsymbol{x}_{0}\right) \rightarrow\left(\boldsymbol{Y}, \boldsymbol{y}_{0}\right)$ of pro $^{*}-\mathrm{HPol}_{\star}$ ranging over the corresponding expansions. The functor $\underline{J}$ induces a faithful functor $J: S h_{\star} \rightarrow S h_{\star}^{*}$, which keeps the object fixed, relating the pointed shape category $S h_{\star}$ with the $S h_{\star}^{*}$. A coarse shape morphism $F^{*}$ is said to be induced provided there exists a shape morphism $F$ such that $F^{*}=J(F)$. A connection between $S h_{\star}^{*}$ and the pointed homotopy category $H T o p_{\star}$ is given by the functor $S^{*}=J \circ S: H T o p_{\star} \rightarrow S h_{\star}^{*}$ where $S$ denotes the shape functor $S: H T o p_{\star} \rightarrow S h_{\star}$.

Recall that, for every $k \in \mathbb{N}$, the functors $\check{\pi}_{k}^{*}: S h_{\star}^{*} \rightarrow \operatorname{Grp}$ and $\check{\pi}_{0}^{*}: S h_{\star}^{*} \rightarrow S e t_{\star}$ ( $S e t_{\star}$ denotes the category of pointed sets and base point preserving functions) associate, with every pointed space $\left(X, x_{0}\right)$, the group $\check{\pi}_{k}^{*}\left(X, x_{0}\right)$ (for $k=0$ the pointed set) called the $k$-th coarse shape group. Its underlaying set is $S h_{\star}^{*}\left(\left(S^{k}, s_{0}\right),\left(X, x_{0}\right)\right)$, i.e., the elements of $\check{\pi}_{k}^{*}\left(X, x_{0}\right)$ are all pointed coarse shape morphisms $A^{*}:\left(S^{k}, s_{0}\right) \rightarrow$ $\left(X, x_{0}\right)$, where $S^{k}$ denotes the standard $k$-dimensional sphere. Notice that a representative of $\left[\left(a_{\lambda}^{n}\right)\right]:\left(S^{k}, s_{0}\right) \rightarrow\left(\left(X_{\lambda}, x_{\lambda}\right), p_{\lambda \lambda^{\prime}}, \Lambda\right)$ of $A^{*}$ consists of $a_{\lambda}^{n} \in \pi_{k}\left(X_{\lambda}, x_{\lambda}\right)$, for all $\lambda \in \Lambda, n \in \mathbb{N}$. The functor $\check{\pi}_{k}^{*}$ associates with every coarse shape morphism $F^{*}:\left(X, x_{0}\right) \rightarrow\left(Y, y_{0}\right)$ a homomorphism (for $k=0$, a base point preserving function) $\check{\pi}_{k}^{*}\left(F^{*}\right): \check{\pi}_{k}^{*}\left(X, x_{0}\right) \rightarrow \check{\pi}_{k}^{*}\left(Y, y_{0}\right)$ given by the following formula:

$$
\check{\pi}_{k}^{*}\left(F^{*}\right)\left(A^{*}\right)=F^{*} \circ A^{*}, A^{*} \in \check{\pi}_{k}^{*}\left(X, x_{0}\right) .
$$

For every induced coarse shape morphism $F^{*}:\left(X, x_{0}\right) \rightarrow\left(Q, q_{0}\right)$ in the category $S h_{\star}^{*}$, where $Q$ is a polyhedron, there exists a unique homotopy class $[f]:\left(X, x_{0}\right) \rightarrow\left(Q, q_{0}\right)$ (a morphism of $H T o p_{\star}$ ) such that $F^{*}=S^{*}([f])$ (see [6] and [5]). Therefore, if we use the abbreviation $\check{\pi}_{k}^{*}(f)$ for the homomorphism $\check{\pi}_{k}^{*}\left(S^{*}([f])\right): \check{\pi}_{k}^{*}\left(X, x_{0}\right) \rightarrow \check{\pi}_{k}^{*}\left(Y, y_{0}\right)$ there is no possibility for ambiguity.

\section{The main result}

Theorem 2.1. Let $\left(X, x_{0}\right)$ be a pointed space and let

$$
\boldsymbol{p}=\left[\left(p_{\lambda}\right)\right]:\left(X, x_{0}\right) \rightarrow\left(\boldsymbol{X}, \boldsymbol{x}_{0}\right)=\left(\left(X_{\lambda}, x_{\lambda}\right), p_{\lambda \lambda^{\prime}}, \Lambda\right)
$$

be an $H$ Pol $_{\star}$-expansion of $\left(X, x_{0}\right)$. Then, for every $k \in \mathbb{N}$,

$$
\check{\pi}_{k}^{*}\left(X, x_{0}\right) \cong \lim _{\leftarrow} \check{\pi}_{k}^{*}\left(\boldsymbol{X}, \boldsymbol{x}_{0}\right),
$$


i.e., the $k$-dimensional coarse shape group of $\left(X, x_{0}\right)$ is isomorphic to the inverse limit of the inverse system $\left(\check{\pi}_{k}^{*}\left(X_{\lambda}, x_{\lambda}\right), \check{\pi}_{k}^{*}\left(p_{\lambda \lambda^{\prime}}\right), \Lambda\right)$ of the $k$-dimensional coarse shape groups of the terms $\left(X_{\lambda}, x_{\lambda}\right)$ in the category Grp. Moreover, the morphism

$$
\check{\pi}_{k}^{*}(\boldsymbol{p})=\left[\left(\check{\pi}_{k}^{*}\left(p_{\lambda}\right)\right)\right]: \check{\pi}_{k}^{*}\left(X, x_{0}\right) \rightarrow\left(\check{\pi}_{k}^{*}\left(X_{\lambda}, x_{\lambda}\right), \check{\pi}_{k}^{*}\left(p_{\lambda \lambda^{\prime}}\right), \Lambda\right)
$$

of pro-Grp induces a group isomorphism

$$
\lim _{\leftarrow} \check{\pi}_{k}^{*}(\boldsymbol{p}): \check{\pi}_{k}^{*}\left(X, x_{0}\right) \rightarrow \lim _{\leftarrow} \check{\pi}_{k}^{*}\left(\boldsymbol{X}, \boldsymbol{x}_{0}\right) .
$$

Proof. Let $k \in \mathbb{N} \cup\{0\}$ and let $G$ denote the inverse limit

$$
G:=\lim _{\leftarrow}\left(\check{\pi}_{k}^{*}\left(X_{\lambda}, x_{\lambda}\right), \check{\pi}_{k}^{*}\left(p_{\lambda \lambda^{\prime}}\right), \Lambda\right)
$$

(in the category $G r p$ ) of the inverse system

$$
\check{\pi}_{k}^{*}\left(\boldsymbol{X}, \boldsymbol{x}_{0}\right)=\left(\check{\pi}_{k}^{*}\left(X_{\lambda}, x_{\lambda}\right), \check{\pi}_{k}^{*}\left(p_{\lambda \lambda^{\prime}}\right), \Lambda\right)
$$

consisting of the coarse shape groups $G_{\lambda}=\check{\pi}_{k}^{*}\left(X_{\lambda}, x_{\lambda}\right)$. Let

$$
A^{*} \in \check{\pi}_{k}^{*}\left(X, x_{0}\right)=S h_{\star}^{*}\left(\left(S^{k}, s_{0}\right),\left(X, x_{0}\right)\right)
$$

be a coarse shape morphism represented by an

$$
\boldsymbol{a}^{*}=\left[\left(a_{\lambda}^{n}\right)\right]:\left(S^{k}, s_{0}\right) \rightarrow\left(\boldsymbol{X}, \boldsymbol{x}_{0}\right) .
$$

Notice that, for every index $\lambda_{0}$, the sequence of homotopy classes:

$$
a_{\lambda_{0}}^{1}, a_{\lambda_{0}}^{2}, \ldots, a_{\lambda_{0}}^{n}, \ldots:\left(S^{k}, s_{0}\right) \rightarrow\left(X_{\lambda_{0}}, x_{\lambda_{0}}\right)
$$

induces an $S^{*}$-morphism which represents a morphism

$$
\boldsymbol{a}_{\lambda_{0}}^{*}:\left(S^{k}, s_{0}\right) \rightarrow\left(X_{\lambda_{0}}, x_{\lambda_{0}}\right)
$$

of pro*$^{*}-\mathrm{HPol}_{\star}$ between rudimentary systems. Now, the coarse shape morphism

$$
A_{\lambda_{0}}^{*}=\left\langle\boldsymbol{a}_{\lambda_{0}}^{*}\right\rangle:\left(S^{k}, s_{0}\right) \rightarrow\left(X_{\lambda_{0}}, x_{\lambda_{0}}\right)
$$

is an element of $G_{\lambda_{0}}$. Further, since $\check{\pi}_{k}^{*}$ is a functor, for every $\lambda \leqslant \lambda^{\prime}$, the homotopy class $p_{\lambda \lambda^{\prime}}:\left(X_{\lambda^{\prime}}, x_{\lambda^{\prime}}\right) \rightarrow\left(X_{\lambda}, x_{\lambda}\right)$ induces a homomorphism $\# p_{\lambda \lambda^{\prime}}:=\tilde{\pi}_{k}^{*}\left(p_{\lambda \lambda^{\prime}}\right): G_{\lambda^{\prime}} \rightarrow$ $G_{\lambda}$. Since, by the property of the $S^{*}$-morphism (1), for every $\lambda \leqslant \lambda^{\prime}$, there exists an $n_{\left(\lambda, \lambda^{\prime}\right)}$ such that

$$
a_{\lambda}^{n^{\prime}}=p_{\lambda \lambda^{\prime}} a_{\lambda^{\prime}}^{n^{\prime}},
$$

for every $n^{\prime} \geqslant n_{\left(\lambda, \lambda^{\prime}\right)}$, the following equivalence of $S^{*}$-morphisms between rudimentary systems holds

$$
\left(a_{\lambda}^{n}\right) \sim\left(p_{\lambda \lambda^{\prime}}^{n}=p_{\lambda \lambda^{\prime}}\right) \circ\left(a_{\lambda^{\prime}}^{n}\right):\left(S^{k}, s_{0}\right) \rightarrow\left(X_{\lambda}, x_{\lambda}\right),
$$

for every pair of fixed indices $\lambda \leqslant \lambda^{\prime}$. This implies that

$$
\boldsymbol{a}_{\lambda}^{*}=\left[\left(p_{\lambda \lambda^{\prime}}^{n}\right)\right] \circ \boldsymbol{a}_{\lambda^{\prime}}^{*}
$$

in pro* $^{*}-\mathrm{HPol}_{\star}$. Consequently, the following equality (of coarse shape morphisms) holds

$$
A_{\lambda}^{*}=S^{*}\left(p_{\lambda \lambda^{\prime}}\right) \circ A_{\lambda^{\prime}}^{*} .
$$

Therefore, $\# p_{\lambda \lambda^{\prime}}\left(A_{\lambda^{\prime}}^{*}\right)=A_{\lambda}^{*}$, which means that $\left(A_{\lambda}^{*} \mid \lambda \in \Lambda\right) \in \prod_{\lambda \in \Lambda} G_{\lambda}$ belongs to the 
group $G=\lim _{\leftarrow}\left(G_{\lambda}, \# p_{\lambda \lambda^{\prime}}, \Lambda\right)$. In such a manner we have defined a function

$$
\Phi: \check{\pi}_{k}^{*}\left(X, x_{0}\right) \rightarrow G
$$

which, to every $A^{*} \in \check{\pi}_{k}^{*}\left(X, x_{0}\right)$, assigns $\Phi\left(A^{*}\right)=\left(A_{\lambda}^{*} \mid \lambda \in \Lambda\right) \in \lim _{\leftarrow}\left(G_{\lambda}, \# p_{\lambda \lambda^{\prime}}, \Lambda\right)$. Notice that $\Phi$ does not depend on the choice of the representative $\boldsymbol{a}^{\leftarrow}$ of the morphism $A^{*}$. Indeed, assuming $\left(a_{\lambda}^{n}\right) \sim\left(a_{\lambda}^{\prime n}\right):\left(S^{k}, s_{0}\right) \rightarrow\left(\boldsymbol{X}, \boldsymbol{x}_{0}\right)$, we infer that, for every $\lambda_{0}$, there exists an $n \in \mathbb{N}$, such that $a_{\lambda_{0}}^{n^{\prime}}=a_{\lambda_{0}}^{\prime n^{\prime}}$, for every $n^{\prime} \geqslant n$. Hence,

$$
\left(a_{\lambda_{0}}^{n}\right) \sim\left(a_{\lambda_{0}}^{\prime n}\right):\left(S^{k}, s_{0}\right) \rightarrow\left(X_{\lambda_{0}}, x_{\lambda_{0}}\right),
$$

which means that both $S^{*}$-morphisms represent the same morphism (2) which is a representative of (3). Thus, $\Phi\left(\left\langle\left[\left(a_{\lambda}^{n}\right)\right]\right\rangle\right)$ and $\Phi\left(\left\langle\left[\left(a_{\lambda}^{\prime n}\right)\right]\right\rangle\right)$ agree at all coordinates.

Let us show that the function $\Phi$ is, actually, the limit homomorphism

$$
\lim _{\leftarrow} \check{\pi}_{k}^{*}(\boldsymbol{p}): \check{\pi}_{k}^{*}\left(X, x_{0}\right) \rightarrow G,
$$

of the morphism

$$
\check{\pi}_{k}^{*}(\boldsymbol{p})=\left[\left(\check{\pi}_{k}^{*}\left(p_{\lambda}\right)\right)\right]: \check{\pi}_{k}^{*}\left(X, x_{0}\right) \rightarrow\left(G_{\lambda}, \# p_{\lambda \lambda^{\prime}}, \Lambda\right)
$$

in pro-Grp, which is induced by the homomorphisms

$$
\check{\pi}_{k}^{*}\left(p_{\lambda}\right): \check{\pi}_{k}^{*}\left(X, x_{0}\right) \rightarrow G_{\lambda}, \lambda \in \Lambda .
$$

It is sufficient to check that

$$
\check{\pi}_{k}^{*}\left(p_{\lambda_{0}}\right)=\theta_{\lambda_{0}} \circ \Phi: \check{\pi}_{k}^{*}\left(X, x_{0}\right) \rightarrow G_{\lambda_{0}},
$$

for every $\lambda_{0} \in \Lambda$, where $\theta_{\lambda_{0}}: \prod_{\lambda \in \Lambda} G_{\lambda} \rightarrow G_{\lambda_{0}}$ denotes the projection. Given a $\lambda_{0}$, for an arbitrary $A^{*}=\left\langle\left[\left(a_{\lambda}^{n}\right)\right]\right\rangle \in \check{\pi}_{k}^{*}\left(X, x_{0}\right)$, an element $\check{\pi}_{k}^{*}\left(p_{\lambda_{0}}\right)\left(A^{*}\right)$ of $G_{\lambda_{0}}$ is defined as the coarse shape morphism

$$
S^{*}\left(p_{\lambda_{0}}\right) \circ A^{*}:\left(S^{k}, s_{0}\right) \rightarrow\left(X_{\lambda_{0}}, x_{\lambda_{0}}\right) .
$$

Notice that $S^{*}\left(p_{\lambda_{0}}\right)$ is represented by

$$
\underline{J}\left(\left[\left(1_{X_{\lambda_{0}}}, \iota\right)\right]\right):\left(\boldsymbol{X}, \boldsymbol{x}_{0}\right) \rightarrow\left(X_{\lambda_{0}}, x_{\lambda_{0}}\right),
$$

where the index function $\iota:\left\{\lambda_{0}\right\} \hookrightarrow \Lambda$ is the inclusion. Therefore, the morphism $S^{*}\left(p_{\lambda_{0}}\right) \circ A^{*}$ is represented by $\left[\left(a_{\lambda_{0}}^{n}\right)\right]:\left(S^{k}, s_{0}\right) \rightarrow\left(X_{\lambda_{0}}, x_{\lambda_{0}}\right)$ which is $\boldsymbol{a}_{\lambda_{0}}^{*}$, exactly. Hence,

$$
\check{\pi}_{k}^{*}\left(p_{\lambda_{0}}\right)\left(A^{*}\right)=S^{*}\left(p_{\lambda_{0}}\right) \circ A^{*}=A_{\lambda_{0}}^{*}=\theta_{\lambda_{0}} \circ \Phi\left(A^{*}\right) .
$$

Thus, the homomorphism $\lim _{\leftarrow} \check{\pi}_{k}^{*}(\boldsymbol{p})$ is equal to $\Phi$.

Next we prove that $\Phi$ is a monomorphism. It is sufficient to check that $\Phi\left(A^{*}\right)=0$ implies $A^{*}=0$. Assuming $\Phi\left(A^{*}\right)=0$ we infer that $A_{\lambda}^{*}=0 \in G_{\lambda}$, for every coordinate $\lambda \in \Lambda$. It follows that, for every $\lambda \in \Lambda$, there exists an $n_{\lambda}$, such that $a_{\lambda}^{n^{\prime}}=0$ (a nullhomotopic map), for every $n^{\prime} \geqslant n_{\lambda}$. It follows that the $S^{*}$-morphism $\left(a_{\lambda}^{n}\right):\left(S^{k}, s_{0}\right) \rightarrow$ $\left(\boldsymbol{X}, \boldsymbol{x}_{0}\right)$ is equivalent to the trivial one. Thus $A^{*}=\left\langle\left[\left(a_{\lambda}^{n}\right)\right]\right\rangle=0$.

In order to prove that $\Phi$ is an epimorphism, suppose that $\left(A_{\lambda}^{*} \mid \lambda \in \Lambda\right)$ is an arbitrary element of the group $G=\lim _{\leftarrow}\left(G_{\lambda}\right.$, \# $\left.p_{\lambda \lambda^{\prime}}, \Lambda\right)$. For every $\lambda_{0} \in \Lambda$, let 
$\left(a_{\lambda_{0}}^{n}\right):\left(S^{k}, s_{0}\right) \rightarrow\left(X_{\lambda_{0}}, x_{\lambda_{0}}\right)$ be an $S^{*}$-morphism such that $\left[\left(a_{\lambda_{0}}^{n}\right)\right]$ represents $A_{\lambda_{0}}^{*}$. Since, for every $\lambda \leqslant \lambda^{\prime}, A_{\lambda}^{*}=\# p_{\lambda \lambda^{\prime}} A_{\lambda^{\prime}}^{*}$, it follows that

$$
\boldsymbol{a}_{\lambda}^{*}=\left[\left(p_{\lambda \lambda^{\prime}}^{n}=p_{\lambda \lambda^{\prime}}\right)\right] \circ \boldsymbol{a}_{\lambda^{\prime}}^{*} .
$$

This implies that there exists an $n_{\left(\lambda, \lambda^{\prime}\right)} \in \mathbb{N}$ such that

$$
a_{\lambda}^{n^{\prime}}=p_{\lambda \lambda^{\prime}} \circ a_{\lambda^{\prime}}^{n^{\prime}}
$$

for every $n \geqslant n_{\left(\lambda, \lambda^{\prime}\right)}$. Therefore, $\left(a_{\lambda}^{n}\right):\left(S^{k}, s_{0}\right) \rightarrow\left(\left(X_{\lambda}, x_{\lambda}\right), p_{\lambda \lambda^{\prime}}, \Lambda\right)$ is an $S^{*}$-morphism such that

$$
\Phi\left(\left\langle\left[\left(a_{\lambda}^{n}\right)\right]\right\rangle\right)=\left(A_{\lambda}^{*} \mid \lambda \in \Lambda\right) .
$$

This completes the proof.

Remark 2.2. Since the category of pointed sets $S e t_{\star}$ admits inverse limits of inverse systems, Theorem 2.1 also holds for the dimension $k=0$. Indeed, it is routine to check that the proof remains valid if we replace groups by pointed sets and homomorphisms by base point preserving functions.

\section{An application}

If $\boldsymbol{p}=\left[\left(p_{\lambda}\right)\right]:\left(X, x_{0}\right) \rightarrow\left(\left(X_{\lambda}, x_{\lambda}\right), p_{\lambda \lambda^{\prime}}, \Lambda\right)$ is the inverse limit of an inverse system of pointed compact polyhedra in the category $T o p_{\star}$, then, by Theorem 9, I.5.3 of $[6]$, the morphism $H \boldsymbol{p}=\left[\left(\left[p_{\lambda}\right]\right)\right]:\left(X, x_{0}\right) \rightarrow\left(\left(X_{\lambda}, x_{\lambda}\right),\left[p_{\lambda \lambda^{\prime}}\right], \Lambda\right)$ of pro-HTop × $_{\star}$ is an $\mathrm{HPol}_{\star}$-expansion of the pointed compact Hausdorff space $\left(X, x_{0}\right)$. Therefore, an immediate consequence of Theorem 2.1, is the property of the continuity of the coarse shape group functor given in the following corollary.

Corollary 3.1. Let $\left(\boldsymbol{X}, \boldsymbol{x}_{0}\right)=\left(\left(X_{\lambda}, x_{\lambda}\right), p_{\lambda \lambda^{\prime}}, \Lambda\right)$ be an inverse system of pointed

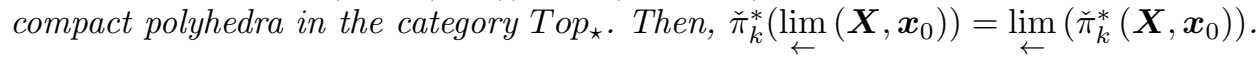

Example 3.2. Let us consider an inverse sequence of groups $\left(G_{i}, q_{i i+1}\right)$, where, for every $i \in \mathbb{N}, G_{i}=\left(\prod_{n \in \mathbb{N}} \mathbb{Z}\right) /\left(\bigoplus_{n \in \mathbb{N}} \mathbb{Z}\right)$ and $q_{i i+1}: G_{i+1} \rightarrow G_{i}$ is the homomorphism $q_{i i+1}(g)=p_{i} \cdot g, g \in G_{i+1}$, induced by the multiplication by some prime number $p_{i} \in$ $\mathbb{N}$. Then, the inverse limit

$$
\lim _{\leftarrow}\left(G_{i}, q_{i i+1}\right)
$$

is equal, up to an isomorphism, to the group

$$
\left(\bigcup_{\left(j_{i}\right) \in \mathbb{N}^{\mathbb{N}}} \prod_{i \in \mathbb{N}}\left(p_{0} \cdots \cdots p_{i-1} \cdot \mathbb{Z}\right)^{j_{i}}\right) / \sim,
$$

where $\sim$ is the equivalence relation "to be equal at all but finitely many coordinates", $\mathbb{N}^{\mathbb{N}}$ denotes the set of all sequences in $\mathbb{N}$ and $p_{0}=1$. Indeed, since $\lim _{\leftarrow}\left(G_{i}, q_{i i+1}\right)$ is, by Corollary 3.1, equal to the coarse shape group $\check{\pi}_{1}^{*}\left(\Sigma_{\left(p_{i}\right)}, x\right)$ of a pointed solenoid $\Sigma_{\left(p_{i}\right)}$, given by a sequence of prime numbers $\left(p_{i}\right)$, for any base point $x \in \Sigma_{\left(p_{i}\right)}$, the conclusion follows by $[\mathbf{1}$, Section 3$]$. 
The following corollary gives a characterization of the triviality of a (sequential) homotopy pro-group via the triviality of the inverse limit of the corresponding sequence of coarse shape groups.

Corollary 3.3. Let $\left(\boldsymbol{X}, \boldsymbol{x}_{0}\right)=\left(\left(X_{i}, x_{i}\right), p_{i i+1}\right)$ be an inverse sequence of pointed compact polyhedra in the category Top . Then

$$
\text { pro- } \pi_{k}\left(\lim _{\leftarrow}\left(\boldsymbol{X}, \boldsymbol{x}_{0}\right)\right) \cong \mathbf{0} \quad \text { if and only if } \lim _{\leftarrow}\left(\check{\pi}_{k}^{*}\left(\boldsymbol{X}, \boldsymbol{x}_{0}\right)\right)=0 \text {. }
$$

More precisely, the homotopy pro-group $\left(\pi_{k}\left(X_{i}, x_{i}\right), \pi_{k}\left(p_{i i+1}\right)\right)$ is trivial in the category pro-Grp if and only if the inverse limit of the group sequence

$$
\left(\left(\prod_{n \in \mathbb{N}} \pi_{k}\left(X_{i}, x_{i}\right)\right) /\left(\bigoplus_{n \in \mathbb{N}} \pi_{k}\left(X_{i}, x_{i}\right)\right),\left[\prod_{n \in \mathbb{N}} \pi_{k}\left(p_{i i+1}\right)\right]\right)
$$

is trivial, where $\left[\prod_{n \in \mathbb{N}} \pi_{k}\left(p_{i i+1}\right)\right]$ is the homomorphism between quotient groups induced by $\pi_{k}\left(p_{i i+1}\right)$.

Proof. By Theorem 4 of [3], for every pointed space $\left(X, x_{0}\right)$ admitting a sequential $H \mathrm{Pol}_{\star}$-expansion, the triviality of its homotopy pro-group pro- $\pi_{k}\left(X, x_{0}\right)$ is equivalent to triviality of its coarse shape group $\check{\pi}_{k}^{*}\left(X, x_{0}\right)$. Now, we can take the pointed space $\lim _{\leftarrow}\left(\boldsymbol{X}, \boldsymbol{x}_{0}\right)$ and apply Corollary 3.1 and the formula for computing the coarse shape group for a polyhedron [3, Example 1].

\section{References}

[1] N. Koceić Bilan, Computing coarse shape groups of solenoids, Math. Commun. 14 (2014) 243-251.

[2] N. Koceić Bilan, On exactness of the coarse shape groups, Glas. Mat. 47 (67) (2012) 209-225.

[3] N. Koceić Bilan, The coarse shape groups, Topology Appl. 157 (2010) 894-901.

[4] N. Koceić Bilan, N. Uglešić, The Whitehead type theorems in coarse shape theory, Homology Homotopy Appl. 15 (2) (2013) 103-125.

[5] N. Koceić Bilan, N. Uglešić, The coarse shape, Glas. Mat. 42 (62) (2007) 145187.

[6] S. Mardešić, J. Segal, Shape Theory, North-Holland, Amsterdam, 1982.

[7] N. Uglešić, Continuity in the coarse and weak shape categories, Mediterr. J. Math. 9 (2012) 741-766.

Nikola Koceić Bilan koceic@pmfst.hr

Department of Mathematics, Faculty of Science, University of Split, Ruđera Boškovića 33, 21000 Split, Croatia 